\title{
Editorial
}

\section{Dynamics, Operator Theory, and Infinite Holomorphy}

\author{
Alfred Peris, ${ }^{1}$ Miguel Martín, ${ }^{2}$ Manuel Maestre, ${ }^{3}$ and Yun Sung Choi ${ }^{4}$ \\ ${ }^{1}$ Departamento de Matemática Aplicada, IUMPA, Universitat Politècnica de València, Edifici 7A, 46022 València, Spain \\ ${ }^{2}$ Departamento de Análisis Matemático, Facultad de Ciencias, Universidad de Granada, 18071 Granada, Spain \\ ${ }^{3}$ Departamento D’Anàlisi Matemàtica, Universitat de València, C/Dr. Moliner 50, 46100 Burjassot, València, Spain \\ ${ }^{4}$ Department of Mathematics, Pohang University of Science and Technology, Pohang 790-784, Republic of Korea
}

Correspondence should be addressed to Alfred Peris; aperis@mat.upv.es

Received 10 July 2014; Accepted 10 July 2014; Published 4 August 2014

Copyright (C) 2014 Alfred Peris et al. This is an open access article distributed under the Creative Commons Attribution License, which permits unrestricted use, distribution, and reproduction in any medium, provided the original work is properly cited.

The works on linear dynamics in the last two decades show that many, even quite natural, linear dynamical systems exhibit wild behaviour. Linear chaos and hypercyclicity have been at the crossroads of several areas of mathematics. More recently, fascinating new connections have started to be explored: operators on spaces of analytic functions, semigroups and applications to partial differential equations, complex dynamics, and ergodic theory.

Related aspects of functional analysis are the study of linear operators on Banach spaces by using geometric, topological, and algebraic techniques, the works on the geometry of Banach spaces and Banach algebras, and the study of the geometry of a Banach space via the behaviour of some of its operators.

In recent years some aspects of the theory of infinitedimensional complex analysis have attracted the attention of several researchers. One is in the general field of Banach and Fréchet algebras and Banach spaces of polynomial and holomorphic functions. Another is in a deep connection with the theory of one and several complex variables as Dirichlet series in one variable, Bohr radii in several variables, Bohnenblust-Hille constants, Sidon constants, domains of convergence, and so forth.

This special issue shows some new advances in the topics shortly described above.

The chaotic behaviour of the annihilation operator $\hat{a}$ of a quantum harmonic oscillator is studied by $\mathrm{X}$. Wu in the following sense. It is shown that the annihilation operator, defined on the Schwartz space of rapidly decreasing functions in $L^{2}(\mathbb{R})$, admits for any $0<\varepsilon<2$ an invariant linear manifold $M$ (i.e., a vector subspace) consisting of vectors such that every pair $(x, y)$ of distinct points in $M$ is $\varepsilon$ distributionally scrambled.

The notion of hypercyclicity (i.e., the existence of dense orbits) is thoroughly studied in recent years for linear operators. J. Bès and J. A. Conejero introduce a new notion of orbit for $N$-linear operators, which is alternative to the one given by K. Grosse-Erdmann and S. K. Kim for bilinear operators and which is inspired by difference equations. Under this new notion, they show that every separable infinite dimensional Fréchet space supports supercyclic $N$-linear operators, for each $N \geq 2$. For the bilinear case, they study the spaces of entire functions and the countable product of the scalar field, and they show that there are bilinear operators with residual sets of hypercyclic vectors.

A generalization of the notion of hypercyclic operator is the concept of convex-cyclic operator, introduced by $\mathrm{H}$. Rezaei, which amounts to require the existence of a vector such that the convex hull generated by its orbit is dense in the space. F. León-Saavedra and M. del Pilar Romero-de la Rosa provide an example of a convex-cyclic operator $T$ such that the power $T^{n}$ fails to be convex-cyclic, which turns out to solve some open questions in the topic.

In the setting of infinite dimensional analysis one important field has been the study of Banach or Fréchet algebras of holomorphic functions. Intensive study has been made on the differential structure of the spectrum of holomorphic functions of bounded type. Another point of view, trying to obtain theorems of Banach-Stone type, has been made to study the homomorphisms between two spaces of holomorphic functions of bounded type for two different complex Banach spaces $X$ and $Y$. V. Dimant et al. have focused on the study of the differential structure of $\mathscr{M}\left(\mathscr{H}_{b}(X), \mathscr{H}_{b}(Y)\right)$, 
the whole set of those homomorphisms. They prove that if $X$ is a symmetrically regular Banach space, then this set has a structure of Riemann domain over $\mathscr{L}\left(X^{*}, Y^{*}\right)$. They also study the size of the fibers.

The failure of Bishop-Phelps theorem for operators between two arbitrary Banach spaces made J. Lindenstrauss, 1963, prove that the set of all operators such that their extension to the biduals attains the norm is always dense in the space of all bounded operators. This, in turn, motivated the study of the same situation for multilinear mappings. That problem was solved positively in 2006. But, given a $n$ multilinear mapping $B$, there are up to $n$ ! different extensions of $B$ to the product of the biduals. J. Falco et al. were able to prove that, for the space $\ell_{1}$, for a prescribed subset $M$ of the permutations of $n$ elements, it is possible to build a $n$-linear form $B$ such that the subset of extensions of $B$ that attains their norm is exactly the one associated with $M$.

The Bishop-Phelps-Bollobás property for numerical radius, a quantitative way of studying numerial radius attaining operators, is studied by S. K. Kim et al.. Among other results, they show that arbitrary $L_{1}(\mu)$ spaces have this property. On the other hand, it is shown that every infinitedimensional Banach space can be renormed to fail the Bishop-Phelps-Bollobás property for numerical radius, while it was known that the Radon-Nikodým property is sufficient for the density of numerical radius attaining operators.

The problem of the asymptotic behaviors of the eigenvalues of a class of Schrödinger operators is related to lowenergy spectral analysis for them. S. Fassari and M. Klaus have studied the asymptotic behavior of the eigenvalues of Schrödinger operator with periodic potential; $X$. Jia et al. have studied the asymptotic behavior of the smallest eigenvalues of Schrödinger operator with potential of critical decay and continue to consider the asymptotic behavior of all other eigenvalues. They obtain the leading terms of discrete eigenvalues when the eigenvalues tend to 0 .

C. Fernández et al. show that localization operators in the discrete setting can be used to obtain information on a signal from the knowledge of the support of its short time Fourier transform. They characterize, in particular, the extremal functions of the uncertainty principle for the discrete short time Fourier transform.

Given a Banach space $X$ and a bounded linear operator $T$ on $X$, J. Koliha et al. obtained various classical results which relate conditions on the spectrum $\sigma(T)$ of $T$ to the operator norm convergence of certain sequences of operators generators generated by T. A. A. Albanese et al. extend them to the class of all Fréchet spaces referred to as prequojections, which can be applied to establish various mean ergodic theorems for bounded operators acting in such Fréchet spaces.

In conclusion, we hope that these papers will enrich our readers and stimulate researchers to extend, generalize, and apply the established results.

\footnotetext{
Alfred Peris Miguel Martín Manuel Maestre Yun Sung Choi
} 


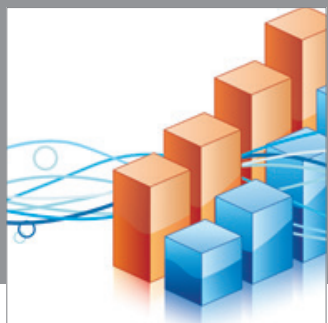

Advances in

Operations Research

mansans

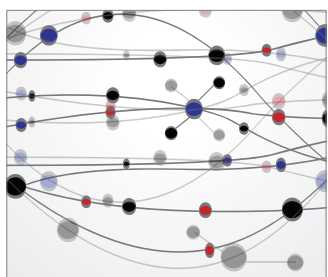

The Scientific World Journal
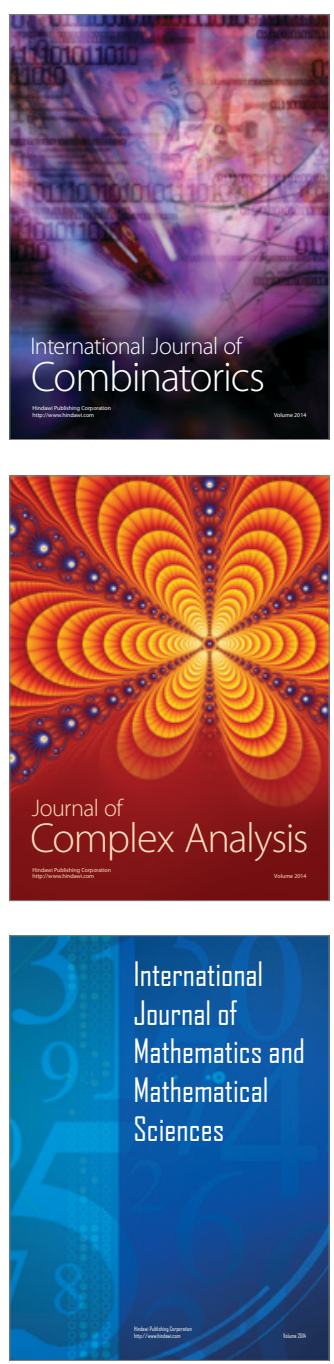
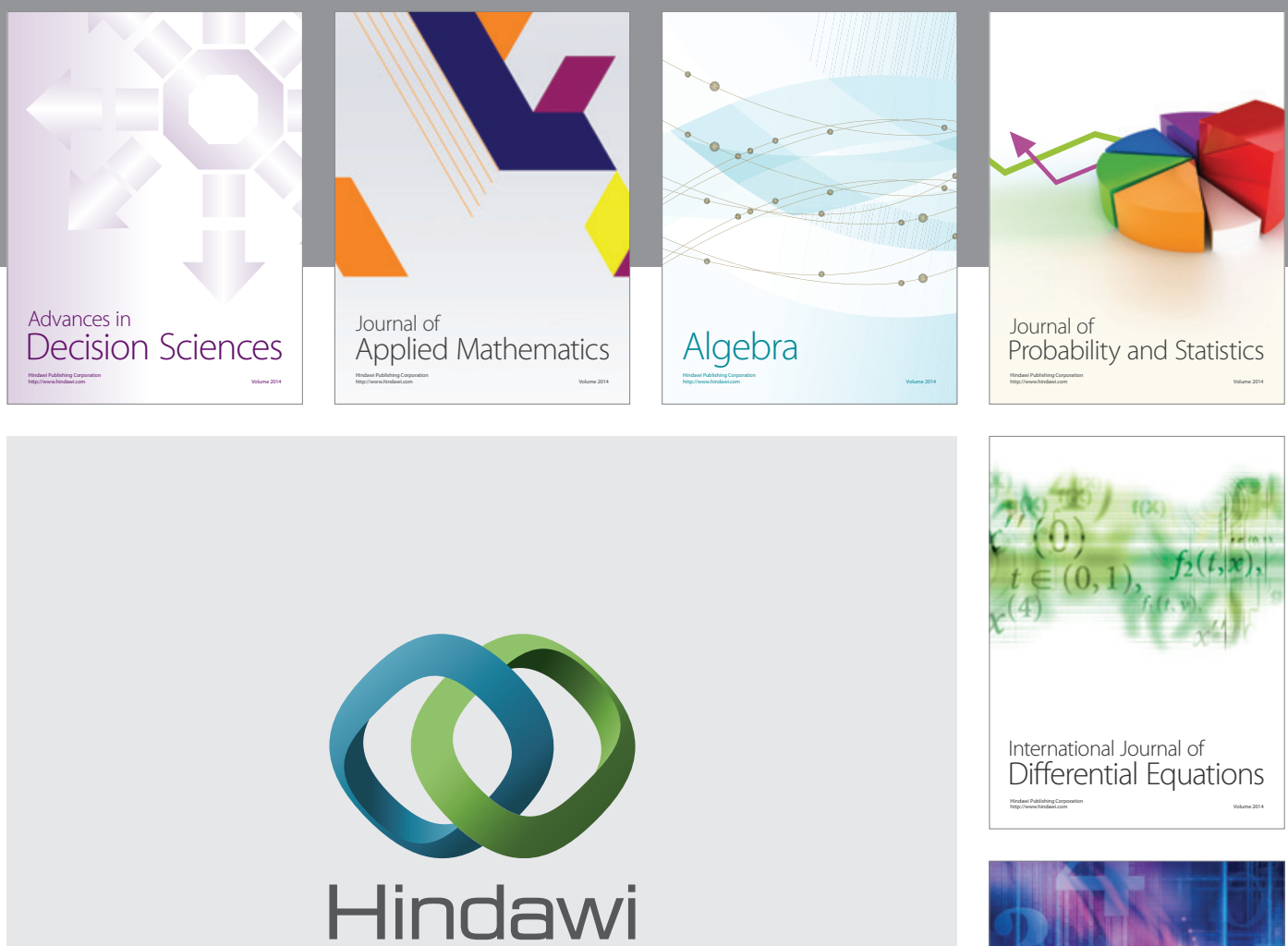

Submit your manuscripts at http://www.hindawi.com
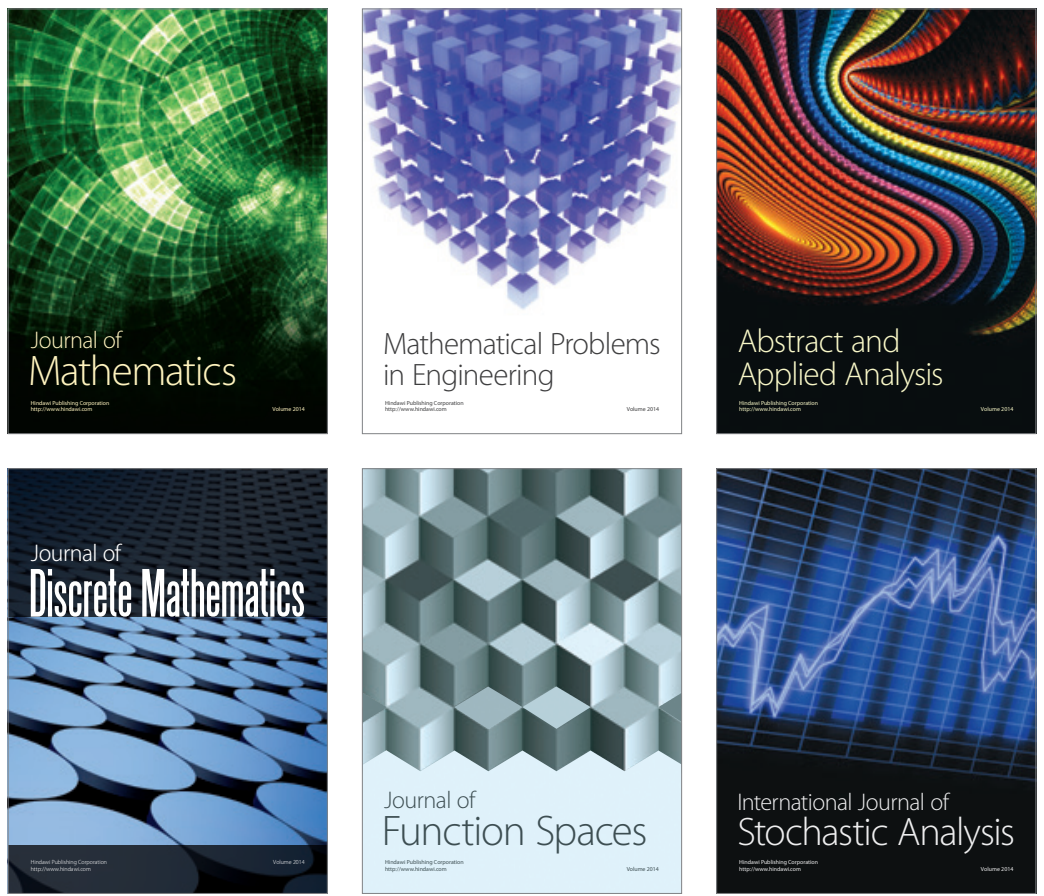

Journal of

Function Spaces

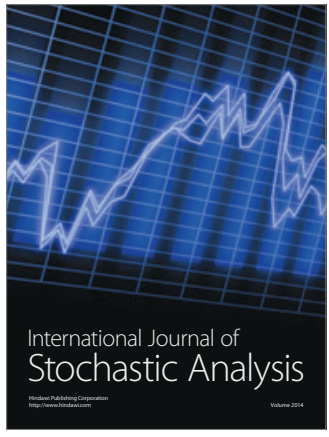

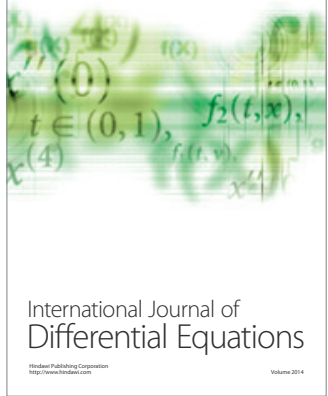
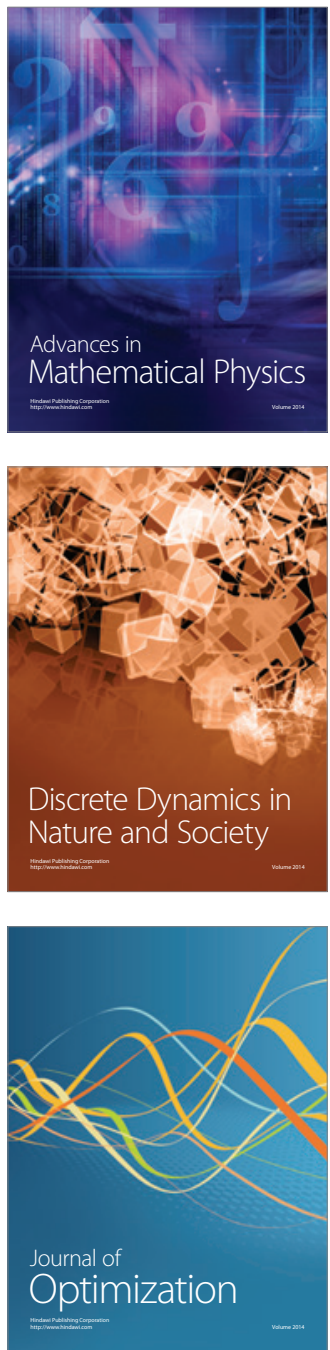